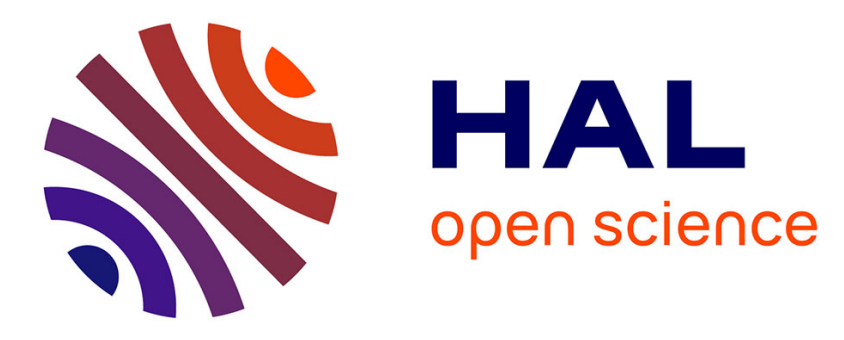

\title{
Output-feedback formation tracking of second-order multi-agent systems with asynchronous variable sampled data
}

Syed Ali Ajwad, Emmanuel Moulay, Michael Defoort, Tomas Menard, Patrick

Coirault

\section{To cite this version:}

Syed Ali Ajwad, Emmanuel Moulay, Michael Defoort, Tomas Menard, Patrick Coirault. Outputfeedback formation tracking of second-order multi-agent systems with asynchronous variable sampled data. 2019 IEEE 58th Conference on Decision and Control (CDC), Dec 2019, Nice, France. pp.44834488, 10.1109/CDC40024.2019.9029375 . hal-02508376

\section{HAL Id: hal-02508376 \\ https://hal.science/hal-02508376}

Submitted on 3 Feb 2021

HAL is a multi-disciplinary open access archive for the deposit and dissemination of scientific research documents, whether they are published or not. The documents may come from teaching and research institutions in France or abroad, or from public or private research centers.
L'archive ouverte pluridisciplinaire HAL, est destinée au dépôt et à la diffusion de documents scientifiques de niveau recherche, publiés ou non, émanant des établissements d'enseignement et de recherche français ou étrangers, des laboratoires publics ou privés. 


\title{
Output-feedback formation tracking of second-order multi-agent systems with asynchronous variable sampled data
}

\author{
Syed Ali Ajwad ${ }^{1}$, Emmanuel Moulay ${ }^{2}$, Michael Defoort ${ }^{3}$, Tomas Ménard ${ }^{4}$, Patrick Coirault ${ }^{1}$
}

\begin{abstract}
This paper deals with the problem of time-varying formation tracking for second-order multi-agent systems under directed topology, where the follower states form a desired formation while tracking the state of the leader. It is considered that each agent, including the leader, has second-order dynamics and can only transmit its position to its neighbors. The velocities and inputs are not exchanged between neighboring agents. In this work, it should be mentioned that contrary to many existing schemes, asynchronous and aperiodic sampling is considered. For each agent, an observer is proposed to estimate its state and the state of its neighbors from the available local asynchronous and aperiodic sampled position data. Using these estimates, a time-varying formation tracking protocol is developed. The stability of the closed-loop system which combines the continuous-discrete time observer and the formation tracking controller is analysed using an appropriate Lyapunov function. The effectiveness of the proposed outputfeedback controller is illustrated for various formations through simulation results.
\end{abstract}

Index Terms-Formation tracking, directed topology, timevarying formation, sampled-data control, asynchronous sampling, continuous-discrete time observer.

\section{INTRODUCTION}

Multi-Agent Systems (MAS) have gained much attention in the last decades due to their application in a vast range of areas such as exploration, defence and rescue to name a few. MAS have also attracted the attention of researchers because of the theoretical challenges that arise in their control and coordination. Mostly, such challenges crop up while controlling the MAS in the absence of any centralized coordination mechanism. Distributed control, based on locally available information, provides much better performance for MAS in terms of scalability, efficiency and reliability.

Formation control has been one of the most attractive/appealing topics within the domain of MAS. It has a broad potential of applications in various areas like surveillance, heavy payload transportation, telecommunication relay, cooperative localization and environmental monitoring [1]. For the formation tracking problem, the followers have to form a desired geometric shape which could be time-varying

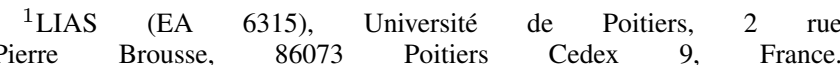
syed.ali.ajwad@univ-poitiers.fr,

patrick.coiraulteuniv-poitiers.fr

${ }^{2}$ XLIM (UMR CNRS 7252), Université de Poitiers, 11 bd Marie et Pierre Curie, 86073 Poitiers Cedex 9, France. emmanuel.moulay@univ-poitiers.fr

${ }^{3}$ Univ. Polytechnique Hauts-de-France, LAMIH, CNRS UMR 8201, Valenciennes 59313, France. michael.defoorteuphf.fr

${ }^{4}$ LAC (EA 7478), Université de Caen, 6 Bd du Marchal Juin, 14032 Caen Cedex, France. tomas.menardeunicaen. fr
}

while the whole formation tracks the trajectory generated by a leader (virtual or not).

Different formation control algorithms have been proposed and can be generally categorized as virtual structure method, leader-follower approach and behaviour based approach [2], [3]. Furthermore, many results have been proposed to derive consensus algorithms for MAS which provide distributed cooperation among the agents to reach some common state using only local information interactions [4], [5], [6]. Consensus protocols can be applied to achieve formation tracking by choosing appropriate states on which the consensus is reached. Such a control technique is considered more reliable and robust as compared to other approaches especially in the case of failure of some agents [7]. Furthermore, in [8], it has been shown that the earlier mentioned approaches can be incorporated in the consensus based ones.

In [9], a consensus protocol has been proposed to solve the finite-time formation tracking problem, with stationary and moving formations. A distributed formation control law for second-order multi-agent systems has been discussed in [10]. Although there exist many approaches to solve the formation tracking problem, only a few works consider the timevarying formation tracking problem. In [11], a formation control algorithm has been proposed for second-order MAS with directed topology and time varying delays. In [12], the case of time-invariant and time-varying finite-time formation tracking problem has been studied. Other examples of timevarying formation protocols can been found in [13], [14], [15].

In the above discussed works, it is generally assumed that each agent's information state is continuously available to its neighbors. However, in practice, continuous measurement and transmission of the whole state is a difficult and costly procedure. Furthermore, due to the digital nature of the onboard equipment, each agent only receives asynchronous and aperiodic sampled position data from its neighbors. Among the different methods to reduce the microprocessor and network loads while guaranteeing the desired control objectives, event-triggered control (ETC) schemes, (where sensors send information to the controller when specific events occur), have been widely studied [16]. This technique involves sensors sending information to the controller when only specific events occur. However, most of these schemes require that each agent's information state is continuously available to its neighbors. Recently, the conditions of continuous state availability are relaxed in [17], [18] whereas the agents' states are measurable. This condition has been relaxed in [19] where an appropriate triggering function has 
been designed to update the output.

In the current paper, the objective is not to design an appropriate triggering function such that the formation tracking problem is achieved, but to design an outputfeedback formation tracking controller. Motivated by [20] where the consensus problem for second-order MAS with arbitrary asynchronous and aperiodic sampling periods has been investigated, an observer-based algorithm has been proposed to solve the distributed formation tracking problem of second-order MAS. Compared to the existing works, the contributions are as follows. Firstly, this paper is a first attempt to deal with time-varying formation tracking problems under aperiodic and asynchronous sampling, where the followers form a desired time-varying geometric shape while the whole formation tracks the trajectory generated by a leader. Secondly, a novel output-feedback controller is proposed. Indeed, it is clear that the results in [20] cannot be directly applied due to the presence of the position and velocity offsets from the desired time-varying geometric shape in the tracking errors. Lastly, a new stability proof of the closed-loop system which combines the continuousdiscrete time observer and the formation tracking controller is derived. Simulations have been carried out for different scenarios (including time-invariant and time-varying formation tracking). The corresponding simulation results have demonstrated the efficiency of the proposed scheme.

The rest of the paper is as follows. In section II, some preliminaries are provided. Section III introduces the formation tracking algorithm along with its stability analysis. Simulation results are presented in section IV while a conclusion is given in section $\mathrm{V}$.

\section{PRELIMINARIES ON GRAPH THEORY}

The communication topology among the agents in a MAS can be described by a directed graph. A directed graph $\mathcal{G}$ is a pair $(\mathcal{V}, \mathcal{E})$, where $\mathcal{V}$ represents a finite nonempty set of nodes and $\mathcal{E} \subseteq \mathcal{V} \times \mathcal{V}$ is a set containing all the edges. An edge is an ordered pair of distinct nodes. For a given edge $(i, j)$ in a directed graph, node $j$ can receive information from node $i$. Therefore, node $j$ is called the child node whereas $i$ is the parent node. A sequence of edges in a directed graph is known as a directed path. A graph has a directed spanning tree if each node has at least one parent node except for one node, which is called the root and has a directed path to all other nodes in the graph. The adjacency matrix $\mathcal{A}=\left(a_{i j}\right) \in \mathbb{R}^{N \times N}$ of $\mathcal{G}$ with $N$ nodes is defined by $a_{i j}=1$ if $(j, i) \in \mathcal{E}$ and $a_{i j}=0$ otherwise. The Laplacian matrix $\mathcal{L} \in \mathbb{R}^{N \times N}$ is defined as $l_{i i}=\sum_{j \neq i} a_{i j}, l_{i j}=-a_{i j}$ for $i \neq j$.

In this paper, the communication connection between the $N$ followers is described by a directed graph $\mathcal{G}$. This is more suitable for various practical applications especially when some agents are equipped with receivers only. Let the diagonal matrix $\mathcal{B}=\operatorname{diag}\left(b_{1}, b_{2}, \ldots, b_{N}\right)$ be the interconnection relationship between the leader and the followers. If follower $i$ can receive information from the leader then $b_{i}=1$ and 0 otherwise. Communication graph including the followers as well as the leader is denoted by $\overline{\mathcal{G}}$. Also

$$
\mathcal{H}=\mathcal{L}+\mathcal{B}
$$

Assumption 1: The pinning joint communication topology $\overline{\mathcal{G}}$ has a directed spanning tree.

The matrix $\mathcal{H}$ is a nonsingular M-matrix if and only if Assumption 1 holds [21]. Furthermore, there exists a diagonal matrix $\Omega=\operatorname{diag}\left(\omega_{1}, \ldots, \omega_{N}\right)$ such that $\mathcal{H}^{T} \Omega+\Omega \mathcal{H}>0$ [22]. Let us denote the smallest eigenvalue of $\mathcal{H}^{T} \Omega+\Omega \mathcal{H}$ as $\rho$ and maximum and minimum eigenvalues of $\Omega$ as $\omega_{\max }$ and $\omega_{\min }$ respectively.

\section{MAIN RESULTS}

\section{A. Problem statement}

Consider a group of $N$ followers labeled from 1 to $N$ and one leader labeled 0 (which could be virtual).

The followers have the following second-order dynamics:

$$
\left\{\begin{array}{l}
\dot{r}_{i}(t)=v_{i}(t), \quad i=1, \ldots, N \\
\dot{v}_{i}(t)=u_{i}(t)
\end{array}\right.
$$

where $r_{i}, v_{i} \in \mathbb{R}^{m}$ represent respectively the position and the velocity of the $i$-th agent and $m \in \mathbb{N}$. The dynamics of the leader is given by:

$$
\left\{\begin{array}{l}
\dot{r}_{0}(t)=v_{0}(t) \\
\dot{v}_{0}(t)=u_{0}(t)
\end{array}\right.
$$

where $r_{0}, v_{0} \in \mathbb{R}^{m}$ represent respectively the position and the velocity of the leader and $m \in \mathbb{N}$.

Assumption 2: The leader input $u_{0}(t)$ is bounded, that is, there exists a constant $\delta_{0} \geq 0$ such that $\left\|u_{0}(t)\right\| \leq \delta_{0}$ for all $t \geq 0$.

In this paper, it is assumed that each agent only measures its position $r_{i}$ (not its velocity $v_{i}$ nor its control input $u_{i}$ ). Furthermore, it is also considered that the agent position is transmitted asynchronously with nonuniform sampling periods. The sampling instant at which the position information is transmitted from agent $j$ to agent $i$ (if there is an edge $(j, i)$ between these two agents) is denoted by $t_{k}^{i, j}$ where $k \in \mathbb{N}$, $i=1, \ldots N$ and $j=0, \ldots N$. Each agent in the network has different sampling instants which are completely independent from all other agents.

Assumption 3: There exists a maximum sampling period $\tau_{M}>0$ such that $0<t_{k+1}^{i, j}-t_{k}^{i, j}<\tau_{M}$, for all $k \in \mathbb{N}$, $i=1, \ldots N$ and $j=0, \ldots N$.

The desired time-varying geometric shape that the followers have to form is defined by $f(t)=\left[f_{1}(t)^{T}, \ldots, f_{N}(t)^{T}\right]^{T}$, where the formation vector $f_{i}(t)=\left[f_{i, r}(t)^{T}, f_{i, v}(t)^{T}\right]^{T}$ for follower $i \in\{1, \ldots, N\}$ satisfies

$$
\dot{f}_{i, r}(t)=f_{i, v}(t)
$$

$f_{i, r}(t), f_{i, v}(t) \in \mathbb{R}^{m}$ corresponds to the position and velocity offset, respectively. It is worth noting that $f_{i}(t)$ does not represent global formation coordinates but relative offset vectors with respect to the leader.

Figure 1 illustrates the notation of formation vector. In this example, the desired geometric shape is a square around 
the leader labeled 0 , in an $x y$-plane i.e. $m=2$. The corresponding formation vectors are $f_{1}=[1,1,0,0]^{T}, f_{2}=$ $[1,-1,0,0]^{T}, f_{3}=[-1,1,0,0]^{T}$ and $f_{4}=[-1,-1,0,0]^{T}$. Since it is a time invariant formation, the corresponding velocity offset components in the formation vectors are zero. Figure 1 provides a graphical representation of the desired formation in the leader frame $\left(x_{0}, y_{0}\right)$.



Fig. 1. Example of square geometric shape.

Assumption 4: It is considered that each agent already has the knowledge of the formation geometry $f(t)$.

Remark 1: The information of formation geometry is provided beforehand to the followers and is not shared during the tracking process.

Definition 1: The formation tracking problem is said to be practically solved if there exists $\bar{\epsilon}>0$ such that

$$
\limsup _{t \rightarrow+\infty}\left\|\left(x_{i}(t)-f_{i}(t)-x_{0}(t)\right)\right\| \leq \bar{\epsilon}
$$

where $x_{i}(t)=\left[r_{i}(t)^{T}, v_{i}(t)^{T}\right]^{T}$ and $x_{0}(t)=$ $\left[r_{0}(t)^{T}, v_{0}(t)^{T}\right]^{T}$.

The objective of this paper is to design the protocol $u_{i}(t)$, $i \in\{1, \ldots, N\}$ such that the MAS (1)-(2) achieves the practical time-varying formation specified in Definition 1 using asynchronous and aperiodic sampled position data.

\section{B. Output-feedback formation tracking controller}

Here, a time-varying formation tracking controller is designed and analyzed. First, for each follower, a continuousdiscrete time observer is proposed to estimate its state and the state of its neighbors from the available local asynchronous and aperiodic sampled position data. Using these estimates, an output-feedback formation tracking protocol is developed.

Similarly to [20], for each follower $i \in\{1, \ldots, N\}$, the following continuous-discrete time observer is used:

$$
\begin{aligned}
& \left.\dot{\hat{r}}_{i, j}(t)=\hat{v}_{i, j}(t)-2 \theta e^{-2 \theta\left(t-t_{k}^{i, j}\right.}\right)\left(\hat{r}_{i, j}\left(t_{k}^{i, j}\right)-r_{j}\left(t_{k}^{i, j}\right)\right)(5) \\
& \dot{\hat{v}}_{i, j}(t)=\dot{f}_{j, v}(t)-\theta^{2} e^{-2 \theta\left(t-t_{k}^{i, j}\right)}\left(\hat{r}_{i, j}\left(t_{k}^{i, j}\right)-r_{j}\left(t_{k}^{i, j}\right)\right)(6)
\end{aligned}
$$

where $j=0,1, \ldots, N, t \in\left[t_{k}^{i, j}, t_{k+1}^{i, j}\right), k \in \mathbb{N}$ and $\theta>0$ is the observer tuning parameter. Moreover, $\dot{f}_{0, v}=0$ as leader does not have any offset. Using the available local asynchronous and aperiodic sampled position data, this observer guarantees the estimation of the state of agent $j$ by agent $i$. $\hat{r}_{i, j}$ and $\hat{v}_{i, j}$ are the estimated position and speed of the agent $j$ by the agent $i$. The initial conditions $\hat{r}_{i, j}(0), \hat{v}_{i, j}(0) \in \mathbb{R}^{m}$ of the observers can be chosen arbitrarily.

Using these estimates, for each follower $i \in\{1, \ldots, N\}$, the formation tracking algorithm is proposed as

$$
\begin{aligned}
& u_{i}(t)=\dot{f}_{i, v}(t) \\
& -\bar{c} \lambda^{2} \sum_{j=1}^{N} a_{i j}\left[\hat{r}_{i, i}(t)-f_{i, r}(t)-\hat{r}_{i, j}(t)+f_{j, r}(t)\right] \\
& -\bar{c} 2 \lambda \sum_{j=1}^{N} a_{i j}\left[\hat{v}_{i, i}(t)-f_{i, v}(t)-\hat{v}_{i, j}(t)+f_{j, v}(t)\right] \\
& -\bar{c} \lambda^{2} b_{i}\left[\hat{r}_{i, i}(t)-f_{i, r}(t)-\hat{r}_{i, 0}(t)\right] \\
& -\bar{c} 2 \lambda b_{i}\left[\hat{v}_{i, i}(t)-f_{i, v}(t)-\hat{v}_{i, 0}(t)\right]
\end{aligned}
$$

where $\bar{c}>0$ is the coupling strength and $\lambda>0$ is the controller tuning parameter.

Remark 2: From Definition 1, the tracking error can be defined as $e_{i}=x_{i}-f_{i}(t)-x_{0}$ for $i \in\{1, \ldots, N\}$. It should be noted that due to the presence of the offset $f_{i}$, the controller (7) is different from the one given in [20]. Therefore, it makes the proof of the closed-loop stability which combines the continuous-discrete time observer and the formation tracking controller relatively complicated.

Theorem 1: Assuming that Assumptions 1-4 are satisfied. There exist constants $\bar{\varrho}>0$ and $\epsilon \in(0,1)$ such that if the observer and controller gains verify

$$
\begin{aligned}
\theta & \leq \frac{\bar{\varrho}}{\tau_{M}} \\
\bar{c} & \geq \frac{\omega_{\max }}{\rho} \\
\lambda & =\epsilon \theta
\end{aligned}
$$

red such that $\lambda \theta>1$, then the time-varying formation tracking problem is solved in the sense of Definition 1 using the output-feedback controller (5)-(7).

Proof: Let us provide a sketch of proof for Theorem 1 inspired from [20]. The agent dynamics can be re-written as

$$
\left\{\begin{array}{l}
\dot{x}_{i}=A x_{i}+B u_{i} \quad i=0, \ldots, N \\
r_{i}=C x_{i}
\end{array}\right.
$$

with $A=\left(\begin{array}{ll}0_{m} & I_{m} \\ 0_{m} & 0_{m}\end{array}\right), B=\left(\begin{array}{c}0_{m} \\ I_{m}\end{array}\right)$ and $C=\left(\begin{array}{ll}I_{m} & 0_{m}\end{array}\right)$. Denoting $\hat{x}_{i, j}=\left(\hat{r}_{i, j}^{T}, \hat{v}_{i, j}^{T}\right)^{T}$, the observer (5)-(6) can be written as

$$
\begin{aligned}
\dot{\hat{x}}_{i, j}(t) & =A \hat{x}_{i, j}(t) \\
& -\theta \Delta_{\theta}^{-1} K_{o} e^{-2 \theta\left(t-\kappa_{i, j}(t)\right)}\left(\hat{r}_{i, j}\left(\kappa_{i, j}(t)\right)-r_{j}\left(\kappa_{i, j}(t)\right)\right)
\end{aligned}
$$

for $i=1, \ldots, N$ and $j=0, \ldots, N$ and where $\kappa_{i, j}(t)=$ $\max \left\{t_{k}^{i, j} \mid t_{k}^{i, j} \leq t, k \in \mathbb{N}\right\}$ is the last instant when the position of agent $j$ has been received by agent $i, \Delta_{\theta}=$ $\left(\begin{array}{cc}I_{m} & 0_{m} \\ 0_{m} & \frac{1}{\theta} I_{m}\end{array}\right), K_{o}=\left[\begin{array}{ll}2 I_{m} & I_{m}\end{array}\right]^{T}$. Similarly, the formation dynamics can be written as

$$
\dot{f}_{i}=A f_{i}+B \dot{f}_{i, v}
$$


Denoting the estimation error $\tilde{x}_{i, j}=\hat{x}_{i, j}-x_{j}$ for $j=0 \ldots N$ and $i=1 \ldots N$, the input $u_{i}$ can be written as

$$
\begin{aligned}
u_{i}= & -\bar{c} K_{c} \Gamma_{\lambda} \sum_{k=1}^{N} \mathcal{H}_{i k} e_{k}-\bar{c} K_{c} \Gamma_{\lambda} \sum_{k=1}^{N} \mathcal{H}_{i k} \tilde{x}_{i, k} \\
& +b_{i} \bar{c} K^{c} \Gamma_{\lambda} \tilde{x}_{i, 0}+B \dot{f}_{i, v}(t)
\end{aligned}
$$

with $K_{c}=\left(\begin{array}{ll}I_{m} & 2 I_{m}\end{array}\right)$ and $\Gamma_{\lambda}=\left(\begin{array}{cc}\lambda^{2} I_{m} & 0_{m} \\ 0_{m} & \lambda I_{m}\end{array}\right)$. Therefore, the formation tracking error dynamics is

$$
\dot{e}_{i}=A e_{i}+B u_{i}-B u_{0}-B \dot{f}_{i, v}
$$

while the estimation error dynamics is

$$
\begin{aligned}
\dot{\tilde{x}}_{i, j}(t)= & \left(A-\theta \Delta_{\theta}^{-1} K_{o} C\right) \tilde{x}_{i, j}(t) \\
& -\theta \Delta_{\theta}^{-1} K_{o} z_{i, j}(t)-B u_{j}(t)+B \dot{f}_{j, v}(t)
\end{aligned}
$$

where $z_{i, j}(t)=\left[e^{-2 \theta\left(t-\kappa_{i, j}(t)\right)} C \tilde{x}_{i, j}\left(\kappa_{i, j}(t)\right)-C \tilde{x}_{i, j}(t)\right]$.

Similarly to $[20]$, and using $\eta^{c}=\left[E_{1}^{T} \ldots E_{N}^{T}\right]^{T}$, $\eta_{i}^{o}=\left[\left(\tilde{X}_{i, 1}\right)^{T} \ldots\left(\tilde{X}_{i, N}\right)^{T}\right]^{T}, i=1, \ldots, N$ and $\eta_{0}^{o}=$ $\left[\tilde{X}_{1,0} \ldots \tilde{X}_{N, 0}\right]$ with $E_{i}=\Gamma_{\lambda} e_{i}$ and $\tilde{X}_{i, j}=\Delta_{\theta} \tilde{x}_{i, j}$, the following Lyapunov functions are selected:

$$
\begin{aligned}
V_{c}\left(\eta^{c}\right) & =\left(\eta^{c}\right)^{T}[\Omega \otimes Q] \eta^{c} \\
V_{o}\left(\tilde{X}_{i, j}\right) & =\left(\tilde{X}_{i, j}\right)^{T} P\left(\tilde{X}_{i, j}\right) \\
\bar{V}_{o}\left(\tilde{X}_{i, j}\right) & =\sum_{i=1}^{N} \sum_{j=0}^{N} s_{i j} V_{o}\left(\tilde{X}_{i, j}\right)
\end{aligned}
$$

where $s_{i j}=1$ if agent $i$ receives information from agent $j$ and 0 otherwise for $i=1, \ldots, N, j=0, \ldots, N, P$ (resp. $Q$ ) is the symmetric positive definite matrix solution of the equation $P+A^{T} P+P A=C^{T} C$ (resp. $Q+Q A+A^{T} Q=$ $\left.Q B B^{T} Q\right)$.

Using these Lyapunov functions, based on conditions (8)(10), one can show that

$$
\sum_{i=1}^{N}\left\|e_{i}(t)\right\| \leq \alpha e^{-\frac{\lambda}{\gamma} t}+\frac{\beta \delta_{0}}{\lambda}, \quad \forall t \geq 0
$$

where $\alpha, \beta, \gamma>0$ and $\delta_{0}$ is the upper bound of the leader input. Furthermore, $\beta$ does not depend on $\theta, \lambda, \bar{c}, \tau_{M}$ and the initial conditions of the agents and observers. Details are omitted due to lack of space.

Remark 3: The conditions (8)-(10) for the selection of the controller and observer gains, obtained from Lyapunov analysis, are only sufficient. It is clear from inequality (14) that the system achieves practical consensus where the tracking error is gradually reduced and enters in a ball centered at the origin. The radius of the ball depends on $\delta$ which means that in case of static leader and time-invariant formation, the tracking errors converge exponentially to zero. Furthermore, the radius of the convergence ball of the tracking errors can be reduced by increasing the controller gain $\lambda$. This will also increase the speed of the controller dynamics.

\section{Simulation Results}

Let us consider a MAS consisting of one leader, labeled 0 , and four followers, labeled $1, \ldots, 4$, described by (1)(2). The communication topology between agents is directed and is shown in Fig. 2. The leader can send its position information only to agent 1 . One can also note that agent 4 can receive data from both agent 1 and agent 3 . The



Fig. 2. Communication topology.

simulation results have been divided into two parts depending on the desired geometric shape which is either time-invariant or time-varying. Different cases for the leader trajectory have also been considered. All the simulations are carried out for 2-dimensional space, i.e. $m=2$. Therefore, position errors are both considered in $x$-position, i.e. $\mid\left(r_{i}\right)_{x}-\left(f_{i, r}\right)_{x}-$ $\left(r_{0}\right)_{x} \mid$ and in $y$-position, i.e. $\left|\left(r_{i}\right)_{y}-\left(f_{i, r}\right)_{y}-\left(r_{0}\right)_{y}\right|$. Fig. 3 shows the sampling instants at which the position information is transmitted between two agents. One can note that the agent position is transmitted asynchronously with nonuniform sampling periods. The maximum sampling period $\tau_{M}$ in these simulations is $130 \mathrm{~ms}$. The observer and controller parameters are chosen as, $\bar{c}=1 \theta=10$ and $\lambda=1.2$.
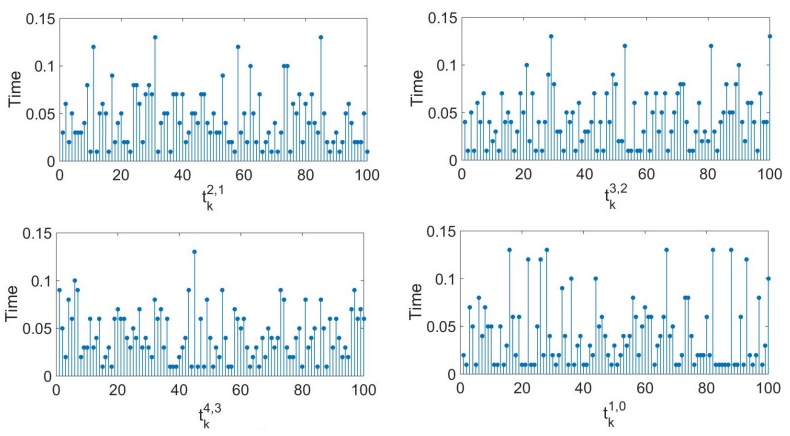

Fig. 3. Sampling periods for data transmission among the agents.

\section{A. Time-invariant formation}

A square geometric shape is considered where four followers track the leader while maintaining a constant square shape around the leader. The formation vector is $f_{1}=$ $[-1,1,0,0]^{T}, f_{2}=[1,1,0,0]^{T}, f_{3}=[1,-1,0,0]^{T}$ and $f_{4}=[-1,-1,0,0]^{T}$.

Case 1: First, it is considered that the leader moves at a constant speed, i.e. $u_{o}=[0,0]^{T}$. The tracking result for 
this case is shown in Fig. 4. Both $x$ and $y$ position errors are depicted in Fig. 5. It is clear from these results that the formation pattern is successfully obtained and all the followers keep the formation while tracking the trajectory of the leader. Moreover, exponential consensus is obtained.

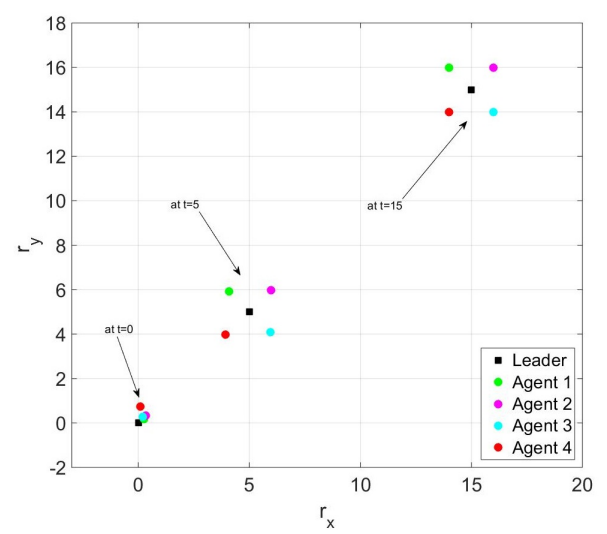

Fig. 4. Time-invariant formation tracking with constant leader velocity
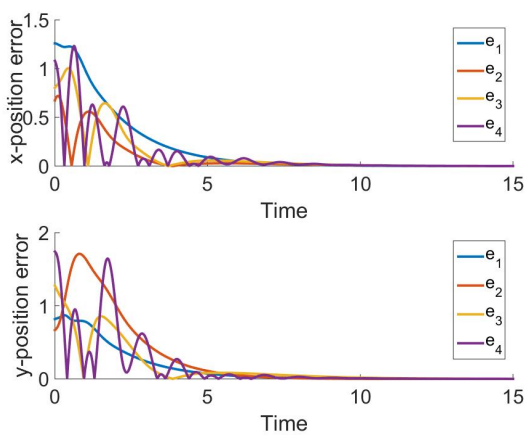

Fig. 5. Position error for constant leader velocity

Case 2: In this case, the leader moves with a constant acceleration, i.e. $u_{0}=[0.1,0.05]^{T}$. The formation tracking result for this case is illustrated in Fig. 6 while Fig. 7 shows the position errors. Practical stability is achieved due to the presence of $u_{0}$ as explained in Remark 3.

\section{B. Time-varying formation}

The time-varying formation vector is

$$
f_{i}(t)=\left(\begin{array}{c}
10 \cos (0.1 t+2 \pi(i-1) / 4) \\
10 \sin (0.1 t+2 \pi(i-1) / 4) \\
-\sin (0.1 t+2 \pi(i-1) / 4) \\
\cos (0.1 t+2 \pi(i-1) / 4)
\end{array}\right)
$$

with $i=1,2,3,4$. If this formation is achieved, the followers will turn in a circle around the leader. Two cases will be considered.

Case 1: In this first case, the leader is static while the followers turn in a circle around the leader. Fig. 8 shows the time-varying formation tracking while Fig. 9 represents the position error.

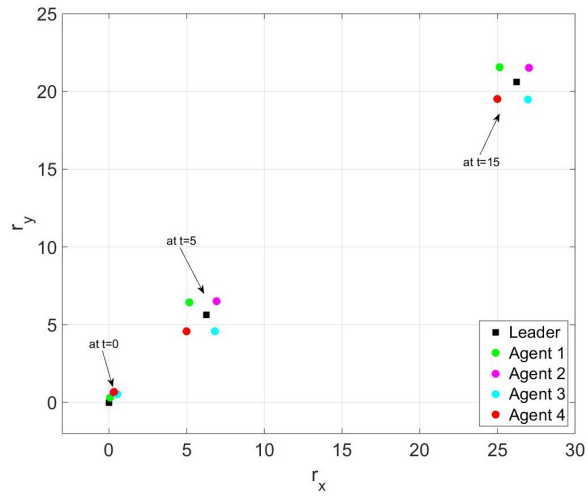

Fig. 6. Time-invariant formation tracking with leader input
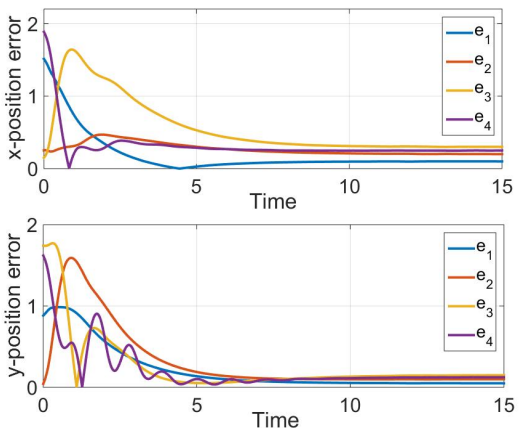

Fig. 7. Position error with leader input

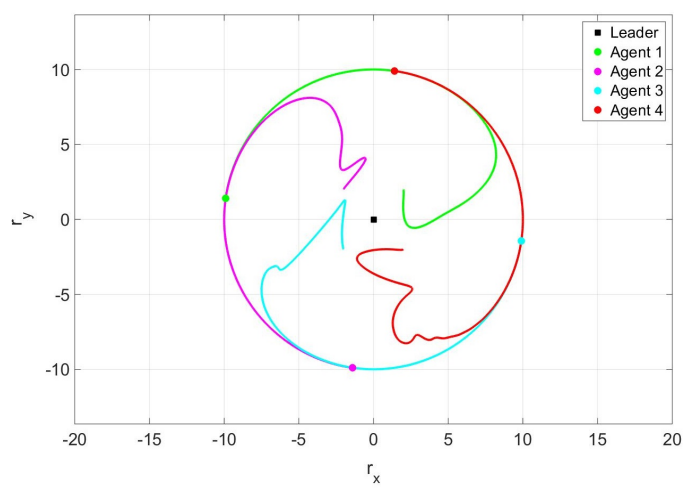

Fig. 8. Time-varying formation tracking with static leader

Case 2: In the second case, the leader has the input $u_{0}=$ $[0.1,0.05]^{T}$. Position trajectories and corresponding position errors are illustrated in Fig. 10 and 11 respectively. Followers not only maintain a circular formation shape but also track the leader. If the leader moves with some constant acceleration, only practical stability is achieved.

\section{CONClusion}

In this paper, the time-varying formation tracking problem for second-order multi-agent systems under some communication constraints has been investigated. The considered communication constraints are the non availability of velocity (and acceleration) information and the transmission 

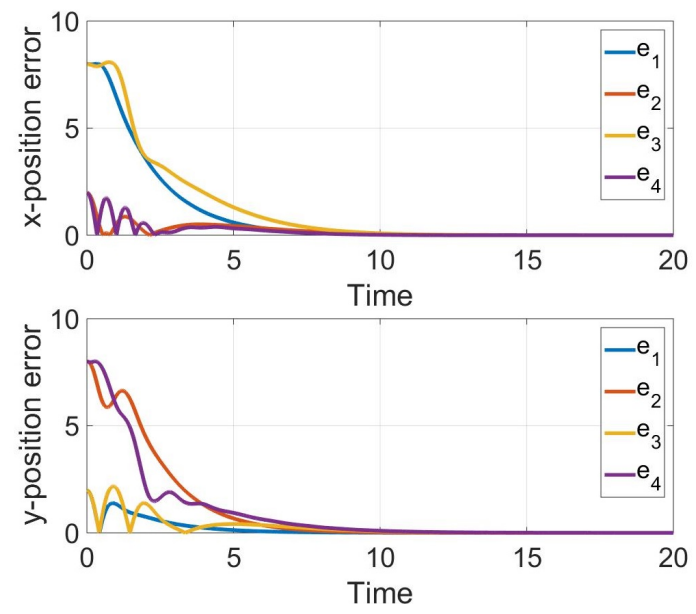

Fig. 9. Position error for time-varying formation with static leader

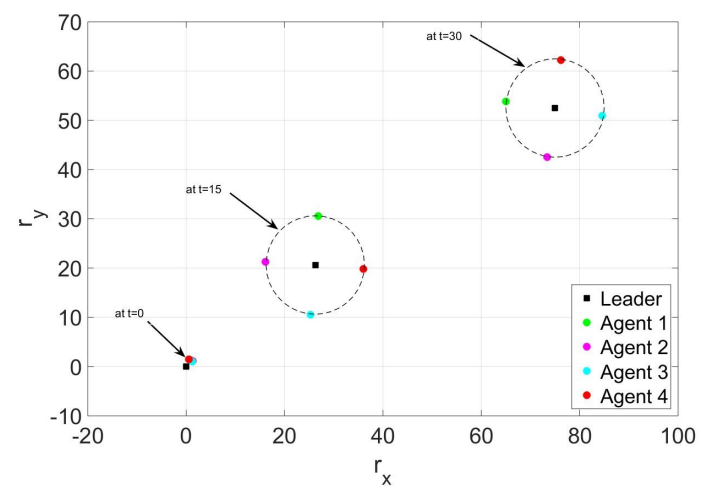

Fig. 10. Time-varying formation tracking with leader input


Fig. 11. Position error for time-varying formation tracking with leader input

of position data at asynchronously and irregular time instants. The followers track the leader while maintaining a desired geometric shape. It has been shown that the proposed observer based algorithm provides an efficient formation tracking in the presence of these communication constraints.

\section{REFERENCES}

[1] W. Wang, J. Huang, C. Wen, and H. Fan, "Distributed adaptive control for consensus tracking with application to formation control of nonholonomic mobile robots," Automatica, vol. 50, no. 4, pp. 12541263,2014

[2] X. Dong and G. Hu, "Time-varying formation control for general linear multi-agent systems with switching directed topologies," $\mathrm{Au}$ tomatica, vol. 73, pp. 47-55, 2016.

[3] W. Ren, "Consensus based formation control strategies for multivehicle systems," in American Control Conference. IEEE, 2006, pp. 4237-4242.

[4] J. Fu, G. Wen, W. Yu, and Z. Ding, "Finite-time consensus for secondorder multi-agent systems with input saturation," IEEE Transactions on Circuits and Systems II: Express Briefs, vol. 65, no. 11, pp. 1758$1762,2018$.

[5] S. Yu and X. Long, "Finite-time consensus for second-order multiagent systems with disturbances by integral sliding mode," Automatica, vol. 54, pp. 158-165, 2015.

[6] P. Lin, W. Ren, C. Yang, and W. Gui, "Distributed consensus of second-order multiagent systems with nonconvex velocity and control input constraints," IEEE Transactions on Automatic Control, vol. 63, no. 4, pp. 1171-1176, 2018.

[7] T. A. Dierks and J. Sarangapani, "Neural network control of mobile robot formations using rise feedback," IEEE Transactions on System, Man and Cybernetics: Part B, vol. 39, no. 2, pp. 332-347, 2009.

[8] W. Ren, "Consensus strategies for cooperative control of vehicle formations," IET Control Theory \& Applications, vol. 1, no. 2, pp 505-512, 2007.

[9] H. Du, S. Li, and X. Lin, "Finite-time formation control of multiagent systems via dynamic output feedback," International Journal of Robust and Nonlinear Control, vol. 23, no. 14, pp. 1609-1628, 2013.

[10] D. Gu and H. Hu, "Distributed network-based formation control," International Journal of Systems Science, vol. 40, no. 5, pp. 539-552, 2009.

[11] X. Lu, F. Austin, and S. Chen, "Formation control for secondorder multi-agent systems with time-varying delays under directed topology," Communications in Nonlinear Science and Numerical Simulation, vol. 17, no. 3, pp. 1382-1391, 2012.

[12] F. Xiao, L. Wang, J. Chen, and Y. Gao, "Finite-time formation control for multi-agent systems," Automatica, vol. 45, no. 11, pp. 2605-2611, 2009.

[13] X. Dong, Y. Zhou, Z. Ren, and Y. Zhong, "Time-varying formation tracking for second-order multi-agent systems subjected to switching topologies with application to quadrotor formation flying," IEEE Transactions on Industrial Electronics, vol. 64, no. 6, pp. 5014-5024, 2017.

[14] X. Dong and G. Hu, "Time-varying formation tracking for linear multiagent systems with multiple leaders," IEEE Transactions on Automatic Control, vol. 62, no. 7, pp. 3658-3664, 2017.

[15] M.-F. Ge, Z.-H. Guan, C. Yang, T. Li, and Y.-W. Wang, "Time-varying formation tracking of multiple manipulators via distributed finite-time control," Neurocomputing, vol. 202, pp. 20-26, 2016.

[16] W. Zhu, Z.-P. Jiang, and G. Feng, "Event-based consensus of multiagent systems with general linear models," Automatica, vol. 50, no. 2 , pp. 552-558, 2014

[17] E. Garcia, Y. Cao, and D. W. Casbeer, "Decentralized event-triggered consensus with general linear dynamics," Automatica, vol. 50, no. 10, pp. 2633-2640, 2014.

[18] V. Dolk, M. Abdelrahim, and W. Heemels, "Event-triggered consensus seeking under non-uniform time-varying delays," IFAC-PapersOnLine, vol. 50, no. 1, pp. 10 096-10 101, 2017, 20th IFAC World Congress.

[19] D. Yang, W. Ren, X. Liu, and W. Chen, "Decentralized eventtriggered consensus for linear multi-agent systems under general directed graphs," Automatica, vol. 69, pp. 242-249, 2016.

[20] T. Ménard, E. Moulay, P. Coirault, and M. Defoort, "Observerbased consensus for second-order multi-agent systems with arbitrary asynchronous and aperiodic sampling periods," Automatica, vol. 99, pp. 237-245, 2019.

[21] Q. Song, F. Liu, J. Cao, and W. Yu, "Pinning-controllability analysis of complex networks: an m-matrix approach," IEEE Transactions on Circuits and Systems I: Regular Papers, vol. 59, no. 11, pp. 26922701, 2012.

[22] H. Zhang, Z. Li, Z. Qu, and F. L. Lewis, "On constructing lyapunov functions for multi-agent systems," Automatica, vol. 58, pp. 39-42, 2015 